\title{
Adaptive Super Twisting Sliding Mode Control With Fractional Order Sliding Surface for Trajectory Tracking of a Mobile Robot
}

Huanhuan Ren ( $\nabla$ custren@163.com )

Changchun University of Science and Technology https://orcid.org/0000-0001-8546-1604

Lizhong Zhang

Changchun University of Science and Technology

Chengzhi Su

Changchun University of Science and Technology

\section{Research Article}

Keywords: fractional order control, super twisting sliding mode control, dynamic control, trajectory tracking, Adaptive control law

Posted Date: March 10th, 2021

DOl: https://doi.org/10.21203/rs.3.rs-229457/v1

License: (c) (i) This work is licensed under a Creative Commons Attribution 4.0 International License. Read Full License 
Adaptive super twisting sliding mode control with fractional order sliding surface for trajectory tracking of a mobile robot

Huanhuan Ren ${ }^{1}$, Lizhong Zhang ${ }^{2}$, Chengzhi Su ${ }^{3}$

College of Mechanical and Electric Engineering, Changchun University of Science and Technology, Changchun, 130022, China

Corresponding author: Lizhong Zhang ${ }^{2}$, custmeen@163.com

\begin{abstract}
This paper presents the position tracking performance of the robot system with uncertainties and external disturbances by using super twisting sliding mode control (STSMC) with fractional order (FO) sliding surface. In this scheme, fractional calculus theory is applied to the design of the sliding surface of STSMC, which can reduce the chattering caused by the switch control action and ensure that the control system has strong robust characteristics and fast convergence. Based on Lyapunov stability theory, the controller ensures the existence of sliding mode of sliding surface in finite time. Moreover, an adaptive STSMC reaching law is adopted. By using the fractional order nonlinear switching manifold and adaptive reaching law, the control performance can be obtained more effectively in sliding mode phase and the reaching phase, respectively. Finally, in order to validate the effectiveness and robustness of the proposed control strategy, the linear PID control strategy and the classical STSMC strategy are designed for comparative analysis, and the numerical calculation is carried out according to the dynamic model to study the position tracking accuracy of the robot under uncertainty and external interference.
\end{abstract}

Keywords: fractional order control; super twisting sliding mode control; dynamic control; trajectory tracking; Adaptive control law

\title{
1. Introduction
}

The application and research of the mobile robot are attracting more and more scholars, and the legged robot is widely studied for its adaptability to various terrain and excellent mobility [1]. Robot needs to control the contact between the foot and the environment to maintain dynamic balance, which is a great challenge to it's stability and the rapid response of the control system [2]. With the progress of computer technology and the development of sensor technology, the application of advanced control theory has significantly improved the performance and ability of the robot system [3]. In this paper, a parallel legged biped robot for carrying heavy objects is designed. Due to the coupling characteristics of the parallel structure and the internal and external interference, the accurate position tracking of the robot becomes a main problem.

Most of the early robot systems used the classical simple controllers. But the walking robot is a complex time-varying dynamic system and vulnerable to environmental interference, the traditional controller cannot meet its high-performance control requirements. Therefore, in order to further improve the control performance of robot system, many advanced control strategies and nonlinear controllers have been proposed and applied, such as predictive control [4], neural network control [5], fuzzy logic control [6], sliding mode control(SMC) [7] and robust control [8]. Among them, SMC has long been considered as an effective and simple special nonlinear control scheme, which can overcome the uncertainty of the system, and has strong robustness to nonsingular external disturbances and unmodeled dynamics of the system, especially for the nonlinear systems [9-11]. In $[12,13]$, SMC strategy based on PID sliding surface was developed to reduce the vibration of 
flexible robot and improve the tracking accuracy of MIMO system, respectively. In [14,15], adaptive SMC and high-order SMC were studied to ensure fast convergence, high control accuracy of the controllers and improve the control effect for uncertain systems.

Because the discontinuous switching function was used in the classical SMC[16], the system state oscillates near the sliding surface instead of sliding along the sliding surface, and the high-frequency chattering can excite the high resonance mode of the system. One of the methods to reduce sliding mode chattering is to use boundary layer control, that is, to replace discontinuous switching function with a saturated continuous control function [17]. Since the boundary of disturbance is variable, it is usually necessary to select a large robust gain to compensate for all disturbances and system uncertainties $[18,19]$. Another way to reduce chattering is to use the high-order sliding mode control. The main idea of this method is to force the discontinuous symbolic function into the time derivative of the control rate, so that the actual control input is continuous through integration operation $[15,16]$ Among them, the super twisting sliding mode control is a simple but effective second-order SMC, which has been studied and applied in many fields [20-22].

Fractional calculus is the extension of traditional integral order differential and integral. The use of fractional calculus theory can more accurately model the controlled object and provide greater flexibility for improving the performance of the controller [23]. And more and more researches have combined fractional calculus with STSMC to further improve the control effect[22,24,25]. In [24], a model free adaptive fractional order super twisting sliding mode control(AFOSTSMC) was proposed to track the robot's trajectory under uncertainties and external disturbances. In [25], an adaptive super twisting fractional order nonsingular terminal sliding mode control (ASTFONTSMC) based on time delay estimation was developed to control the manipulator.

In this paper, a new type of walking robot is taken as the research object, the dynamic model of the robot is established, and the gravity term and Coriolis force term are compensated by the calculated torque controller (CTC). Then, a STSMC based on fractional order sliding surface is designed to realize the closed-loop position control of the robot system. The stability and tracking accuracy of the closed-loop system are improved by the inherent memory and heredity of the fractional order operator. In Sect. 2, this paper introduces the definition and basic properties of fractional calculus. In Sect. 3, the general dynamic model of the robot system is described and the adaptive super twisting sliding mode control with fractional order sliding surface (AFOSTSMC) is proposed while other controllers are designed for comparison. In Sect. 4, the simulation results show the excellent performance of the proposed controller.

2. Definition and principles of fractional calculus

Fractional calculus is a generalization of integral calculus [26], and the expression of continuous fractional calculus is:

$$
D_{t}^{\alpha} f(t)= \begin{cases}\frac{d^{\alpha}}{d t^{\alpha}} f(t), & \operatorname{Re}(\alpha)>0 \\ f(t), & \operatorname{Re}(\alpha)=0 \\ \int_{a}^{t} f(t) d \tau^{-\alpha} & \operatorname{Re}(\alpha)<0\end{cases}
$$

where ${ }_{a} D_{t}^{\alpha}$ is fractional calculus operator; $a$ and $t$ represent the lower and upper bounds of the 
fractional calculus; $\alpha$ is the fractional order, which can be a complex number.

Researchers have defined a variety of forms of fractional calculus, and two kinds of fractional calculus which are widely used in control field are Riemann Liouville (RL) and Caputo definitions

$[22,27]$.The RL fractional calculus of function $f(t)$ is defined as:

$$
{ }_{a}^{R L} D_{t}^{\alpha} f(t)=\frac{1}{\Gamma(n-\alpha)}\left(\frac{d}{d t}\right)^{n} \int_{a}^{t} \frac{f(\tau)}{(t-\tau)^{1+\alpha-n}} d \tau
$$

where $n-1<\alpha<n, n \in N ; \Gamma(\cdot)$ represents Euler's Gamma function and is given by:

$$
\Gamma(z)=\int_{0}^{\infty} e^{-t} t^{z-1} d t
$$

The Caputo's definition can be written as:

$$
{ }_{a}^{C} D_{t}^{\alpha} f(t)=\frac{1}{\Gamma(n-\alpha)} \int_{a}^{t} \frac{f^{n}(\tau)}{(t-\tau)^{1+\alpha-n}} d \tau
$$

where $n-1<\alpha \leq n$.

The $n_{t h}$ order derivative of the fractional order derivative operator ${ }_{a} D_{t}^{\alpha} f(t)$ can be expressed as:

$$
\frac{d^{n}}{d t^{n}}\left({ }_{a} D_{t}^{\alpha} f(t)\right)={ }_{a} D_{t}^{\alpha}\left(\frac{d^{n} f(t)}{d t^{n}}\right)={ }_{a} D_{t}^{\alpha+n} f(t)
$$

For Lyapunov function $V(t)$ with initial value $V\left(t_{0}\right)$, finite-time stability can be computed as[24]:

$$
\dot{V}(t) \leq-n V^{p}(t), \quad \forall t \geq t_{0}, \quad V\left(t_{0}\right) \geq 0
$$

where $n>0$ and $0<p<1$. Therefore, the finite-time $t_{f}$ can be formulated as

$$
t_{f} \leq \frac{V^{1-p}\left(t_{0}\right)}{n(1-p)}
$$

3. Control schemes design

3.1. Description of system dynamics

In order to verify the proposed AFOSTSMC method, the dynamic modeling and analysis of the robot are carried out, equation 8 can be used to describe the dynamics of $n$-DOF(degree of freedom) system:

$$
M(q) \ddot{q}+V(q, \dot{q}) \dot{q}+G(q)+F_{d}=F
$$

where $q, \dot{q}, \ddot{q} \in \mathfrak{R}^{n}$ represent the generalized position, generalized velocity, generalized acceleration, respectively; $M(q) \in \mathfrak{R}^{n \times n}$ is a bounded, symmetric and positive definite inertia matrix; $V(q, \dot{q}) \in \mathfrak{R}^{n}$ represents the Coriolis force and centripetal force; $F_{d} \in \mathfrak{R}^{n}$ represents the 
unknown external disturbances; $G(q) \in \mathfrak{R}^{n}$ and $F \in \mathfrak{R}^{n}$ represent the conservative force and dissipative forces, respectively.

Equation 8 is established with Cartesian coordinate $q$ as the generalized coordinate system, and $F$

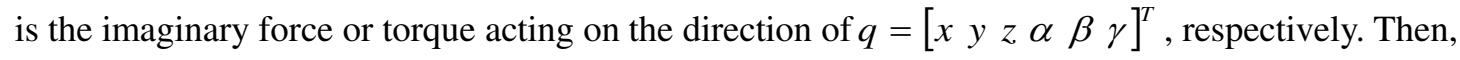
the performance requirements of the controller can be described as follows: a suitable controller designed according to equation 8 can ensure the robot to track the reference trajectory $q$ accurately with the presence of external disturbance $F_{d}$.

\subsection{Control schemes based on calculated torque controller}

PID control is the most widely used liner control method in the research of robot drive system and the whole closed-loop system control. In [28], the design to the implementation of the proportional, integral and derivative (PID) remote control system of robot manipulator using Matlab-based internet network was proposed, which could monitor the operation response of the manipulator remotely in the process of operation. In order to improve the control effect of the robot system, [2930] designed schemes based on the outer loop PID controller and the inner loop calculated torque controller(CTC). As shown in Fig.1, the unknown external disturbance is ignored in this control scheme. The nonlinear force term $V(q, \dot{q})$ and the gravity term $G(q)$ are compensated in the inner loop to realize the decoupling and linearization of the system and make the robot become a more easily controlled system.

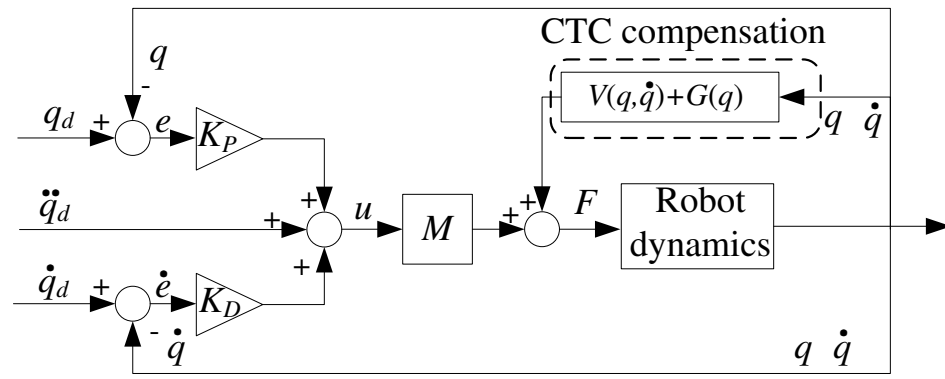

Fig. 1 PD control with CTC

The control rules shown in Fig. 1 are as follows:

$$
\left\{\begin{array}{l}
F=M(q) u+V(q, \dot{q}) \dot{q}+G(q) \\
u=\ddot{q}_{d}+K_{P}\left(q_{d}-q\right)+K_{D}\left(\dot{q}_{d}-\dot{q}\right)
\end{array}\right.
$$

where $q, \dot{q}_{d}, \ddot{q}_{d}$ are the reference position, velocity and acceleration inputs, respectively; $K_{P}, K_{D}$ represent the are the gain of position error and velocity error, respectively; $u$ is the controller output. Submitting equation 8 into equation 9 , one can get:

$$
\ddot{q}=\ddot{q}_{d}+K_{P} e+K_{D} \dot{e}
$$

where $e, \dot{e}$ is the position error and velocity error, respectively. By defining $\ddot{e}=\ddot{q}_{d}-\ddot{q}$, equation 
11 can be obtained:

$$
\ddot{e}+K_{P} e+K_{D} \dot{e}=0
$$

The stability of the closed-loop control system can be easily proved by the following positive definite quadratic Lyapunov functions:

$$
V=\frac{1}{2} \dot{e}^{T} \dot{e}+\frac{1}{2} e^{T} K_{P} e \Rightarrow \dot{V}=-\dot{e}^{T} K_{D} \dot{e} \leq 0
$$

It is obvious that the tracking error $e$ converges to 0 , and different closed-loop performances can be obtained by changing $K_{P}$ and $K_{D}$.

PID controller is a simple linear control scheme, and its control performance is mainly determined by model accuracy. When the model accuracy is high enough and the system is not affected by external interference, the PID control scheme based on CTC can achieve satisfactory results by reasonably selecting $K_{P}$ and $K_{D}$. For complex robot systems, due to the rigid structure dynamics, the flexible dynamics, the driving system dynamics and other structural and non-structural uncertainties, it is difficult to obtain a high-precision model. The convergence region of trajectory tracking error is determined by the modeling error and uncertainty region.

Aiming at the perturbation of model parameters caused by internal and external uncertainties, the sliding mode controller is considered to improve the tracking performance and ensure the robustness and fast convergence of the robot system. Based on the decoupling principle of CTC and without considering the external disturbance $F_{d}$, a super twisting sliding mode controller with CTC is designed according to the traditional sliding surface. The main purpose of developing this method is to reduce the chattering effect.

Firstly, the sliding surface is selected as the following equation:

$$
s=\Lambda e+\dot{e}
$$

where $\Lambda>0$ is the sliding surface matrix, which is a diagonally positive definite matrix; $e$ and $\dot{e}$ are the position error and velocity error vector, respectively.

Substituting the time derivative of $s$ into equation 8 with $F_{d}=0$, one can get:

$$
\dot{s}=\Lambda \dot{e}+\ddot{q}_{d}-M^{-1}(q)[F-C(q, \dot{q}) \dot{q}-G(q)]
$$

Furthermore, the generalized force $F$ can be expressed by equation 15:

$$
F=M(q)\left[\left(\Lambda \dot{e}+\ddot{q}_{d}-\dot{s}\right)\right]+C(q, \dot{q}) \dot{q}+G(q)
$$

where $\dot{s}$ is the STSMC reaching rate, and can be can be defined by the following equation 16 :

$$
\left\{\begin{array}{l}
\dot{s}=-k_{1}|s|^{\frac{1}{2}} \operatorname{sign}(s)+w \\
\dot{w}=-k_{2} \operatorname{sign}(s)
\end{array}\right.
$$

where $k_{1}, k_{2}>0 ; \operatorname{sign}(\cdot)$ represents the sign function.

\subsection{Fractional order STSMC scheme}

Equation 8 can be rewritten as equation 17: 


$$
\bar{M} \ddot{q}+H(q, \dot{q}, \ddot{q})=F
$$

where $\bar{M}$ is the positive diagonal matrix; $H(q, \dot{q}, \ddot{q})=(M(q)-\bar{M}) \ddot{q}+V(q, \dot{q}) \dot{q}+G(q)+F_{d}$ is used to describe all the unknown dynamics of the system, including friction, interference, etc.

In order to further improve the performance of sliding mode control, many researchers applied the fractional order theory to the design of sliding surface or reaching rate[24,31]. The nonlinear fractional order sliding surface can be defined as [33]:

$$
s=\dot{e}+\omega D_{t}^{\varepsilon} \operatorname{sgn}(e)^{\rho}+\eta D_{t}^{\mu-1} \operatorname{sgn}(e)^{v}
$$

where $s=\left(s_{1}, s_{2}, \cdots s_{n}\right)^{T}, \operatorname{sgn}(e)^{v}=|e|^{v} \operatorname{sign}(e)$ with $0<v<1$ and $0<\rho<1, \varepsilon$ and $\mu$ are fractional order ranges between $0<\varepsilon, \mu<1, \eta \in \mathfrak{R}^{n \times n}$ and $\omega \in \mathfrak{R}^{n \times n}$ are positive diagonal matrix. According to equation 5, the following equation can be obtained:

$$
\dot{s}=\ddot{e}+\omega D_{t}^{\varepsilon+1} \operatorname{sgn}(e)^{\rho}+\eta D_{t}^{\mu} \operatorname{sgn}(e)^{v}
$$

Combining equation 19 and equation 17, one can get:

$$
\dot{s}=\ddot{q}_{d}-\bar{M}^{-1}[F-H(q, \dot{q}, \ddot{q})]+\omega D_{t}^{\varepsilon+1}(e)^{\rho}+\eta D_{t}^{\mu} \operatorname{sgn}(e)^{v}
$$

Equation 21 can be obtained by substituting equation 16 into equation 20 :

$$
F=\bar{M}\left[\ddot{q}_{d}+\omega D_{t}^{\varepsilon+1}(e)^{\rho}+\eta D_{t}^{\mu} \operatorname{sgn}(e)+k_{1}|s|^{\frac{1}{2}} \operatorname{sign}(s)+w\right]+H(q, \dot{q}, \ddot{q})
$$

The stability of the closed-loop system is obtained by the following Lyapunov functional candidate [24]:

$V=\zeta^{T} P \zeta=\left(2 k_{2}+\frac{k_{1}^{2}}{2}\right) \zeta_{1}^{2}+\zeta_{2}^{2}-k_{1} \zeta_{1} \zeta_{2}$

where $\zeta=\left[\zeta_{1}, \zeta_{2}\right]^{T}=\left[|s|^{\frac{1}{2}} \operatorname{sign}(s), w\right]^{T}, P=\left[\begin{array}{cc}2 k_{2}+0.5 k_{1}^{2} & -0.5 k_{1} \\ -0.5 k_{1} & 1\end{array}\right]$ is the positive definite symmetric matrix and satisfies equation 30 :

$$
A^{T} P+P A=-Q
$$

where $A=\left[\begin{array}{lll}-0.5 k_{1} & 0.5 ;-k_{2} & 0\end{array}\right]$ is Hurwitz matrix. $Q$ is any positive definite symmetric matrix. According to equation 29, equation 33 can be obtained:

$$
\lambda_{\min }(P)\|\zeta\|^{2} \leq V \leq \lambda \max (P)\|\zeta\|^{2}
$$

where $\lambda_{\min }(P)$ and $\lambda_{\max }(P)$ represent the minimum and maximum eigenvalues of matrix $P$, respectively.

By taking derivative of $\zeta$, equation 25 can be obtained: 


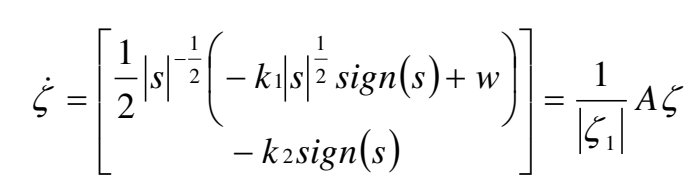

The derivative of equation 22 with respect to time is shown in equation 26.

$$
\dot{V}=\frac{1}{\left|\zeta_{1}\right|} \zeta^{T}\left(A^{T} P+P A\right) \zeta=-\frac{1}{\left|\zeta_{1}\right|} \zeta^{T} Q \zeta
$$

Next, one can get equation 27:

$$
\left|\zeta_{1}\right|=|s|^{\frac{1}{2}} \leq\|\zeta\| \leq \frac{V^{\frac{1}{2}}}{\lambda^{\frac{1}{2}} \min (P)}
$$

Substitution of equation 27 into equation 26, one can derive:

$$
\dot{V} \leq-\frac{1}{\zeta_{1}} \lambda_{\text {min }}(Q)\|\zeta\|^{2}=-\frac{\|\zeta\|}{\left|\zeta_{1}\right|} \lambda_{\text {min }}(Q)\|\zeta\| \leq-\lambda_{\text {min }}(Q)\|\zeta\|
$$

Equation 29 can be obtained from equation 24 :

$$
V^{\frac{1}{2}} \leq \lambda^{\frac{1}{2}} \max (P)\|\zeta\|
$$

Substituting equation 28 into equation 29 yields:

$$
\dot{V} \leq-\frac{\lambda \min (Q)}{\lambda^{\frac{1}{2}} V_{\max }(P)}
$$

It can be obtain that the closed-loop system equation 16 tends to 0 in finite time, and $e \rightarrow 0, \dot{e} \rightarrow 0$ when $t \rightarrow \infty$, so the closed-loop system is asymptotically stable. The nonlinear system will reach the origin along $s=0$ in finite time. The corresponding finite-time can be calculated as equation 31, according to equation 7 :

$$
t_{f} \leq \frac{2 V^{\frac{1}{2}}\left(\zeta_{0}\right) \lambda^{\frac{1}{2}} \text { max }(P)}{\lambda_{\text {min }}(Q)}
$$

This information can be obtained from equations 21 and 31 . The output of the controller and convergence time are related to parameters $k_{1}$ and $k_{2}$ in the reaching rate (equation 16) of STSMC. By changing the gains, the system performance and convergence time can be adjusted.

In the control scheme mentioned above, the system is considered to be known and bounded. However, in practical application, it is not easy to obtain a high-precision model and design a control scheme. At the same time, it is difficult to determine the uncertainty domain, which makes it difficult to select the appropriate parameters $k_{1}$ and $k_{2}$. In order to improve the control performance, the following adaptive super-twisting reaching law (ASTRL) is selected [22]: 


$$
\begin{cases}\dot{k}_{1} & =\left\{\begin{array}{lll}
m \cdot \operatorname{sign}(|s|-n) & \text { if } & k_{1}>\bar{k}_{1} \\
\bar{\mu} & \text { if } & k_{1} \leq \bar{k}_{1}
\end{array}\right. \\
k_{2}=\sigma \cdot k_{1} & \end{cases}
$$

where $m, n, \bar{k}_{1}, \bar{\mu}, \sigma$ are positive coefficients; $\bar{k}_{1}$ and $\bar{\mu}$ are arbitrary small positive coefficients.

The improved adaptive fractional order super twisting sliding mode control (AFOSTSMC) scheme is shown in the Fig. 2.

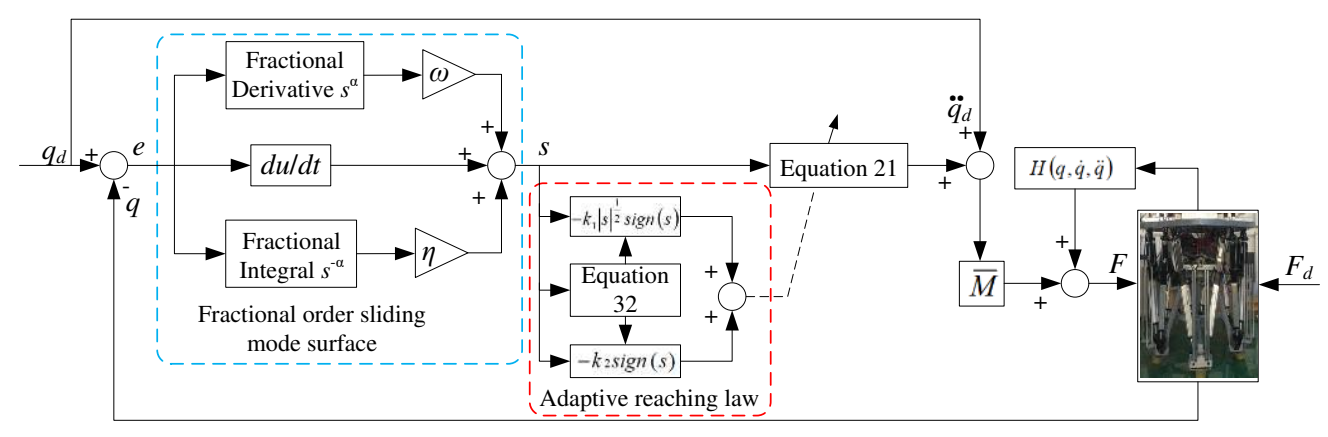

Fig. 2 AFOSTSMC scheme

\section{Comparative simulationss}

In this section, in order to verify the effectiveness of the AFOSTSMC, the simulation work is divided into two parts: one is to compare and analyze the tracking effect of the proposed controllers to the nominal model without external interference; the other is to compare and analyze the tracking effect of the controllers when the robot position is deviated by external disturbance and unmodeled dynamics. The robot is required to move according to the following ideal trajectory:

$$
\operatorname{Tr} a=0.375 t^{3}-0.281 t^{4}+0.056 t^{5}
$$

When moving as trajectory equation 33 , the moving time is $2 \mathrm{~s}$, the moving distance is $0.3 \mathrm{~m}$, and the velocity and acceleration at the start and end time are both 0 .

Firstly, the tracking effects of the controllers to the nominal model are compared and analyzed by selecting reasonable controller parameters: $K_{P}=1.85$ and $K_{D}=75$ in PD-CTC scheme are selected; in STSMC scheme, $\Lambda=20, k_{1}=0.6, k_{2}=1.3$; for AFOSTSMC, sliding surface(equation 18) and adaptive reaching law(equation 32) parameters are chosen as follows, $\omega=2, \eta=10, \mu=0.2, \varepsilon=0.2, \rho=0.8, v=0.6, \bar{\mu}=0.01 m=8, n=0.02, \bar{k}_{1}=0.01$, $\sigma=4$.

The trajectory tracking effect of the three controllers on the nominal model is shown in Fig. 3. IT is the ideal trajectory, PIDT is the tracking trajectory of PD-CTC scheme, STSMCT is the tracking trajectory of STSMC, and AFOSTSMCT is the tracking trajectory of AFOSTSMC scheme. Fig. 3(b) shows the tracking error between the three control schemes and the ideal trajectory, PIDTE, STSMCTE and AFOSTSMCTE represent the position errors of PD-CTC, STSMC and AFOSTSMC, respectively. 


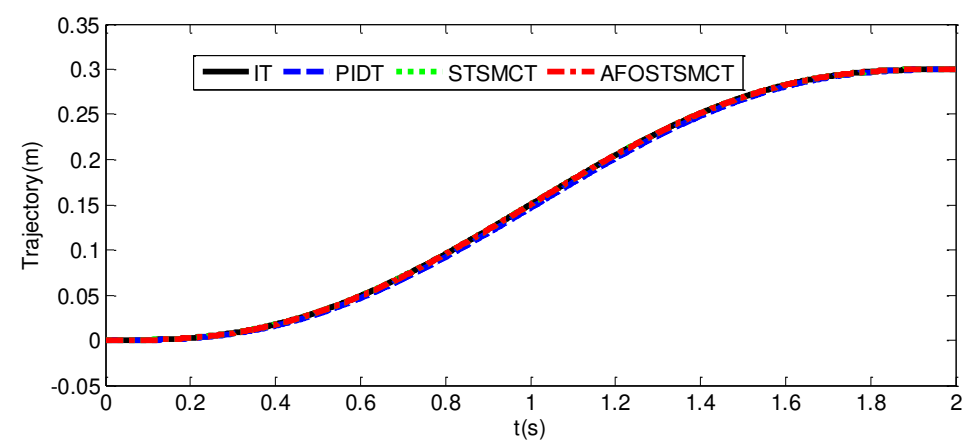

(a)

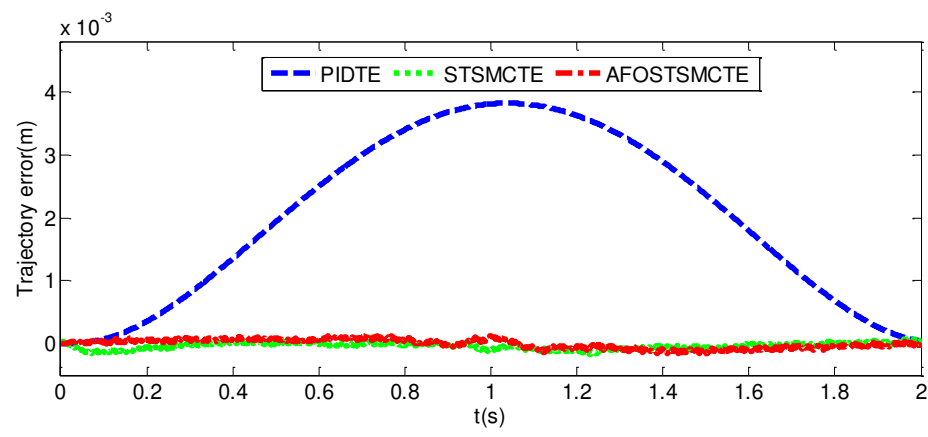

(b)

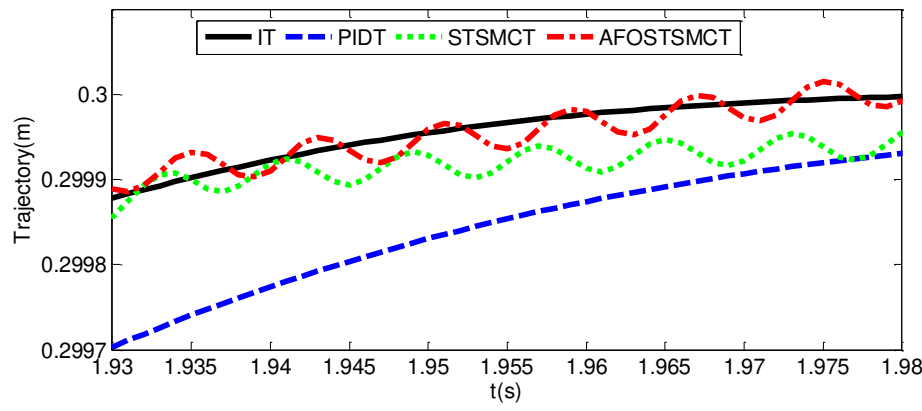

(c)

Fig. 3 Trajectory tracking performance of the three controllers for the nominal model

It can be obtained from Fig. 3(a) that the tracking performances of the three control schemes to the ideal trajectory of the nominal model is excellent, and all of them can accurately track the reference trajectory equation 33. Fig. 3(b) shows that in the process of tracking the input ideal trajectory, the tracking error of PID-CTC scheme is larger than that of the other two schemes. AFOSTSMC and STSMC schemes have faster adjustment speed and smaller error value. In Fig. 3(c), it can be seen that the trajectory can traverse the ideal trajectory continuously and smoothly during the adjustment process of AFOSTSMC scheme. From the perspective of the whole control process, it is clearly concluded that the trajectory tracking performance of the proposed AFOSTSMCT method has better convergence speed and accuracy than PID-CTC and STSMC.

Then, considering the unmodeled factors and the presence of external disturbances, the tracking effects of the three controllers are compared. The position change as shown in Fig.4 is added in the tracking process, where $0-1 \mathrm{~s}$ represents non-periodic disturbance, and1-2s is sinusoidal signal with frequency of $10 \mathrm{rad} / \mathrm{s}$ and amplitude of $5 \mathrm{~mm}$, representing periodic disturbance. 


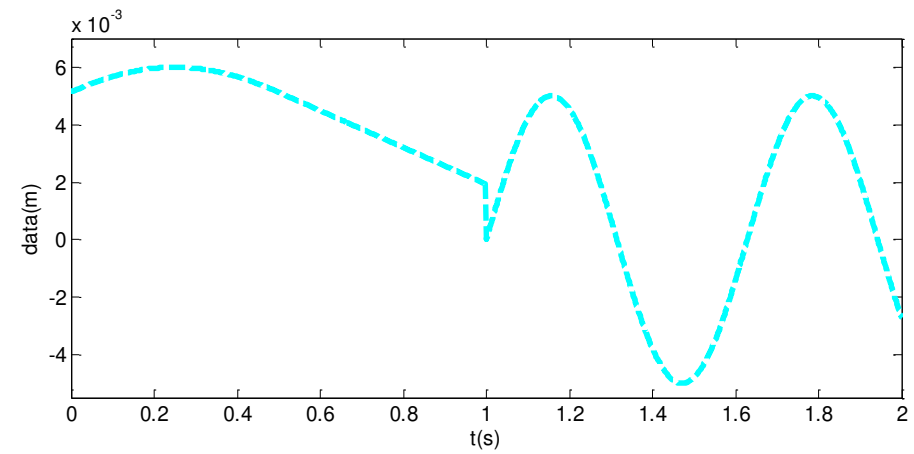

Fig. 4 Position disturbance

In the presence of the position disturbance shown in Fig. 4, the tracking performance of the three controllers to the ideal trajectory is shown in Fig. 5.

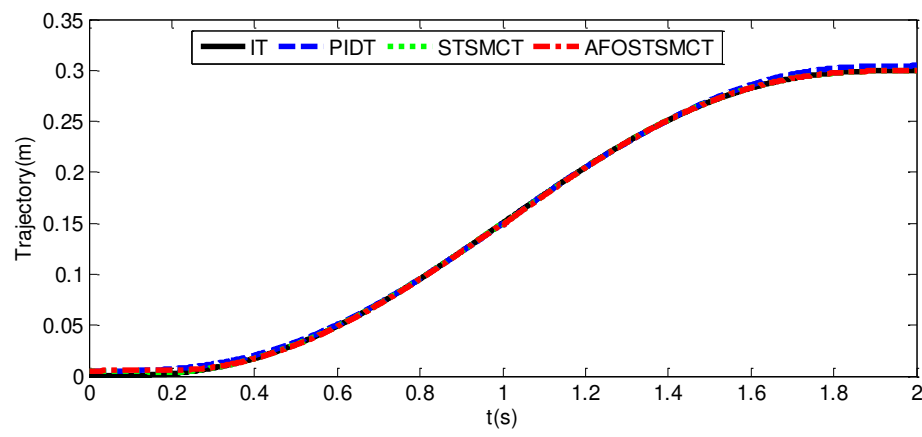

(a)

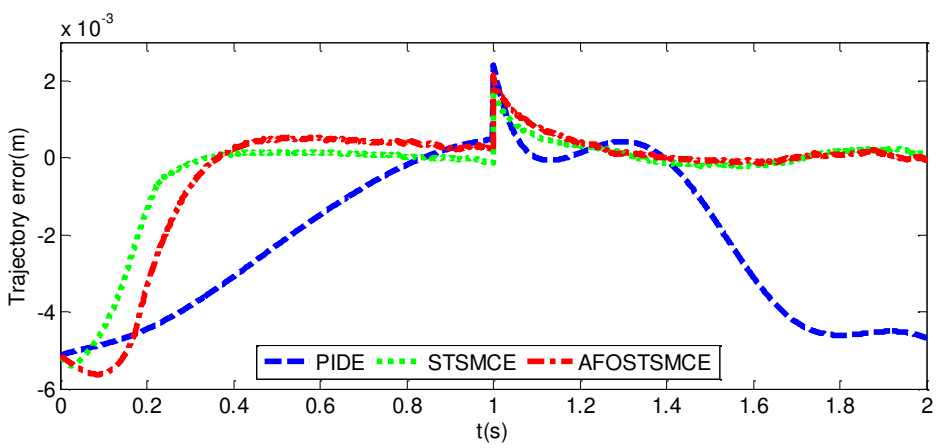

(b)

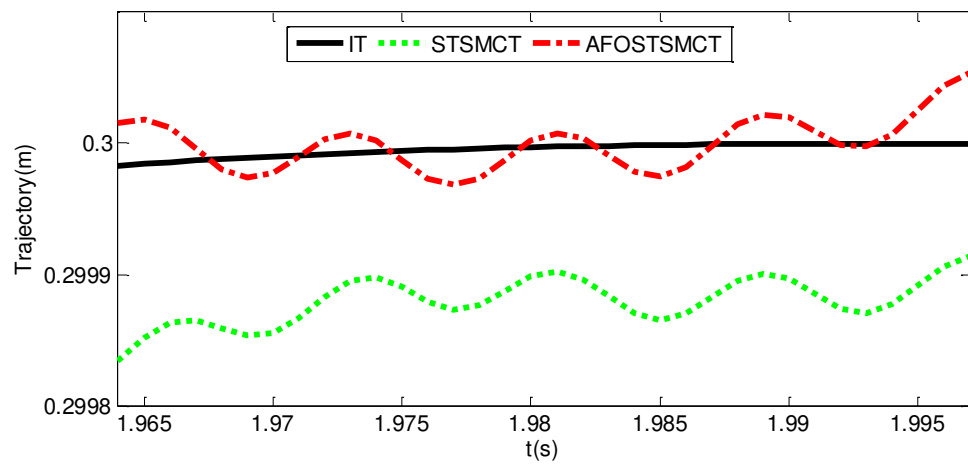

(c) 


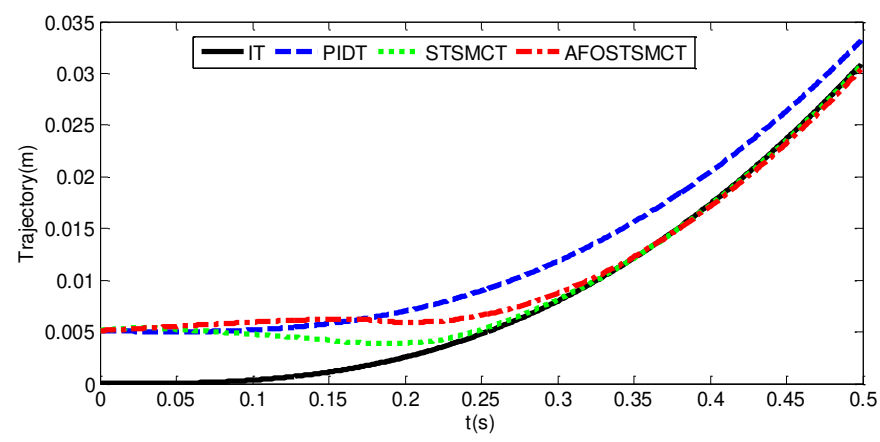

(d)

Fig. 5 Trajectory tracking performance of the three controllers with position disturbance It can be observed from Fig. 5(a) and (d) that PID-CTC, STSMC and AFOSTSMC can track the ideal trajectory in the presence of non-periodic position disturbance and STSMC has the fastest processing speed. Fig. 5(c) and (b) shows that the STSMC and AFOSTSMC can keep trajectory tracking in the presence of periodic disturbance, while the PID-CTC control has large position deviation. In the process of AFOSTSMC scheme adjustment, the tracking trajectory can traverse the ideal trajectory continuously and smoothly.

\section{Conclusion}

In order to solve the problem of uncertain dynamics caused by model parameter perturbation and external disturbance, a super twisting sliding mode control with fractional order sliding surface is designed to track the robot's trajectory. The stability and tracking accuracy of the closed-loop system are improved by the inherent memory and heredity of fractional calculus operator, and the finite time stability of the closed-loop system is established by using Lyapunov function. Furthermore, an adaptive reaching law is designed to improve the performance of the controller. To verify the performance of the AFOSTSMC, simulations are carried out in the presence of position disturbance and without position disturbance respectively, and the classical PID-CTC scheme and STSMC scheme are designed as the comparison. In conclusion, compared with the other two control schemes, AFOSTSMC has better performance in terms of robustness, fast response and tracking error, especially in dealing with periodic disturbances. In addition, the robustness of AFOSTSMC is more obvious when the frequency and amplitude of periodic disturbance increase.

Conflict of Interest: The authors declare that they have no conflict of interest.

Funding: This study was funded by Defense Industrial Technology Development Program OF CHINA (JCKY2019411B001).

\section{References}

[1] Nilsson N. J, “A Mobile Automaton: An Application of Artificial Intelligence Techniques”, 1st International Joint Conference on Artificial Intelligence. Morgan Kaufmann Publishers Inc. 1969.

[2] Faraji S, Razavi H , Ijspeert A J, "Bipedal walking and push recovery with a stepping strategy based on time-projection control", The International Journal of Robotics Research, 2019, 38(5):587-611.

[3] Kim Y B , Seok D Y, Lee S Y, et al., "6-Axis Force/Torque Sensor With a Novel Autonomous Weight Compensating Capability for Robotic Applications”, IEEE Robotics and Automation 
Letters, 2020, 5(4):6686-6693.

[4] [Chen Y, Luo X, Han B, et al., "Model Predictive Control with Integral Compensation for Motion Control of Robot Manipulator in Joint and Task Spaces", IEEE Access, 2020, PP(99):1-1.

[5] Li X, Cheah C C, "Adaptive Neural Network Control of Robot Based on a Unified Objective Bound", IEEE Transactions on Control Systems Technology, 2014, 22(3):1032-1043.

[6] Soliman M A, Azar A T, Saleh M A, et al., "Path Planning Control for 3-Omni Fighting Robot Using PID and Fuzzy Logic Controller", International Conference on Advanced Machine Learning Technologies and Applications, Springer, Cham, 2019.

[7] Su Y, Zheng C, "A new nonsingular integral terminal sliding mode control for robot manipulators", International Journal of Systems Science, 2020(2):1-11.

[8] Bingol M C, Akpolat Z H, Koca G O, "Robust Control of a Robot Arm Using an Optimized PID Controller”, International Conference Mechatronics, Springer, Cham, 2017.

[9] Ying-Jeh H, Tzu-Chun K, Shin-Hung C, "Adaptive Sliding-Mode Control for Nonlinear Systems With Uncertain Parameters”, IEEE Trans. Syst., 2009.

[10] Chin C S, Lin W P, "Robust Genetic Algorithm and Fuzzy Inference Mechanism Embedded in Sliding-Mode Controller for Uncertain Underwater Robot", IEEE/ASME Transactions on Mechatronics, 2018:1-1.

[11] Su Y, Zheng C, "A new nonsingular integral terminal sliding mode control for robot manipulators", International Journal of Systems Science, 2020(2):1-11.

[12] Khan A H, Li S, "Sliding Mode Control With PID Sliding Surface for Active Vibration Damping of Pneumatically Actuated Soft Robots", IEEE Access, 2020, PP(99):1-1.

[13] Sunila M S, Sankaranarayanan V, Sundereswaran K, "Optimised sliding mode control for MIMO uncertain non-linear system with mismatched disturbances", Electronics Letters, 2018, 54(5):290-291.

[14] Qi W, Zong G, Karim H R, “Observer-Based Adaptive SMC for Nonlinear Uncertain Singular Semi-Markov Jump Systems With Applications to DC Motor”, IEEE Transactions on Circuits and Systems I: Regular Papers, 2018:2951-2960.

[15] Ahmed S, Wang H, Tian Y, "Adaptive High-Order Terminal Sliding Mode Control Based on Time Delay Estimation for the Robotic Manipulators With Backlash Hysteresis", IEEE Transactions on Systems, Man, and Cybernetics: Systems, 2019:1-10.

[16] Cao Y, Chen X B, “An Output-Tracking-Based Discrete PID-Sliding Mode Control for MIMO Systems”, IEEE/ASME Transactions on Mechatronics, 2014, 19(4):1183-1194.

[17] Young D K, Utkin V I, Ozguner U, “A control engineer's guide to sliding mode control”, 1996 IEEE International Workshop on. IEEE, 2002.

[18] [Sun, Jianming, "Chattering-Free Sliding-Mode Variable Structure Control of Delta Operator System”, Journal of Computational and Theoretical Nanoscience, 2014, 11(10):2150-2156.

[19] Chang J L, Tsai J C, "Dynamic Output Feedback Sliding Mode Controller Design for Chattering Avoidance", Journal of Marine ence and Technology, 2014, 22(3):277-284.

[20] Guo L, Wang H, Jin N, et al., "A Speed Sensorless Control Method for Permanent Magnet Synchronous Motor Based on Super-Twisting Sliding Mode Observer”, 2019 14th IEEE Conference on Industrial Electronics and Applications (ICIEA). IEEE, 2019.

[21] Jinli X, Zhen P, "Super-twisting sliding mode control of permanent magnet synchronous motor based on voltage compensation”, Journal of Physics: Conference Series, 2020, 1605(1):012010 
(6pp).

[22] Gao P, Zhang G, Ouyang H, et al., "An Adaptive Super Twisting Nonlinear Fractional Order PID Sliding Mode Control of Permanent Magnet Synchronous Motor Speed Regulation System Based on Extended State Observer", IEEE Access, 2020, PP(99):1-1.

[23] Tavazoei M S, Haeri M, Jafari S, et al., "Some Applications of Fractional Calculus in Suppression of Chaotic Oscillations", IEEE Transactions on Industrial Electronics, 2008, 55(11):4094-4101.

[24] Ahmed S, Ahmed A, Mansoor I, et al., "Output Feedback Adaptive Fractional-Order SuperTwisting Sliding Mode Control of Robotic Manipulator", Iranian Journal of Science and Technology - Transactions of Electrical Engineering, 2020:1-13.

[25] Yaoyao Wang, Jiawang Chen, “Adaptive super-twisting fractional-order nonsingular terminal sliding mode control of cable-driven manipulators", ISA Transactions, 2018, 75:163-180.

[26] Momeni H R, Dadras S, "Fractional-order dynamic output feedback sliding mode control design of robust stabilization of uncertain fractional-order nonlinear systems", Asian Journal of Control: Affiliated with ACPA, the Asian Control Professors' Association, 2014.

[27] Delavari H, Ghaderi R, Ranjbar A, et al., " Fuzzy fractional order sliding mode controller for nonlinear systems", Communications in Nonlinear ence \& Numerical Simulation, 2010, 15(4):963-978.

[28] Khairudin M, "PID Control for a Manipulator Robot Using Internet Networking and Matlabbased", Journal of Physics Conference Series, 2018, 1140.

[29] Zhang X, Liu J, Gao Q, et al., "Adaptive robust decoupling control of multi-arm space robots using time-delay estimation technique", Nonlinear Dynamics, 2020(1).

[30] Van M, Kang H J, Suh Y S, et al., "Output feedback tracking control of uncertain robot manipulators via higher-order sliding-mode observer and fuzzy compensator", Journal of Mechanical ence \& Technology, 2013, 27(8):2487-2496.

[31] Haitao L, Khalil H K, "Output feedback stabilization using super-twisting control and highgain observer", International Journal of Robust and Nonlinear Control, 2019, 29. 
Figures

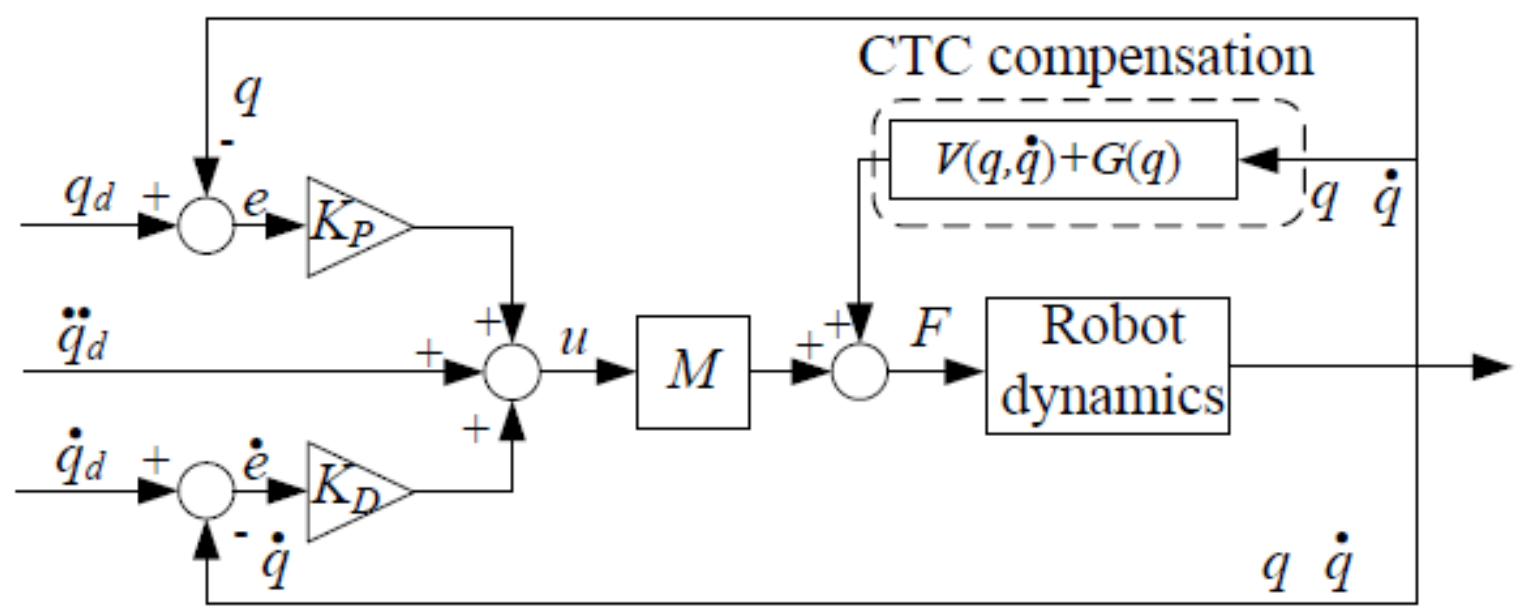

Figure 1

PD control with CTC

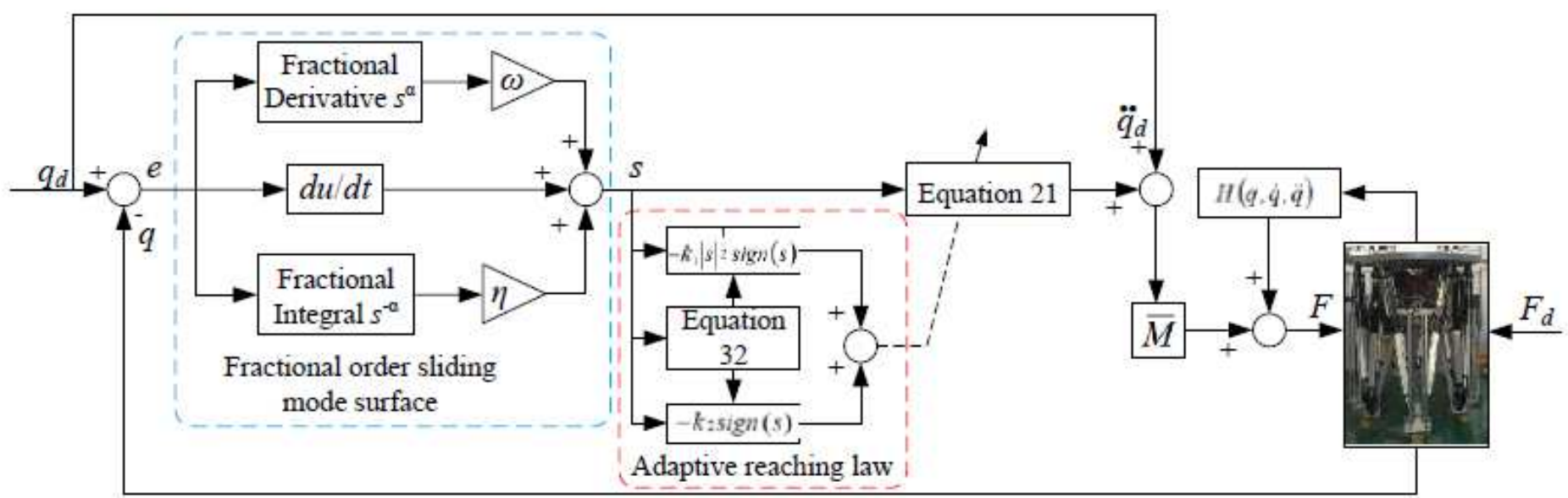

Figure 2

AFOSTSMC scheme 


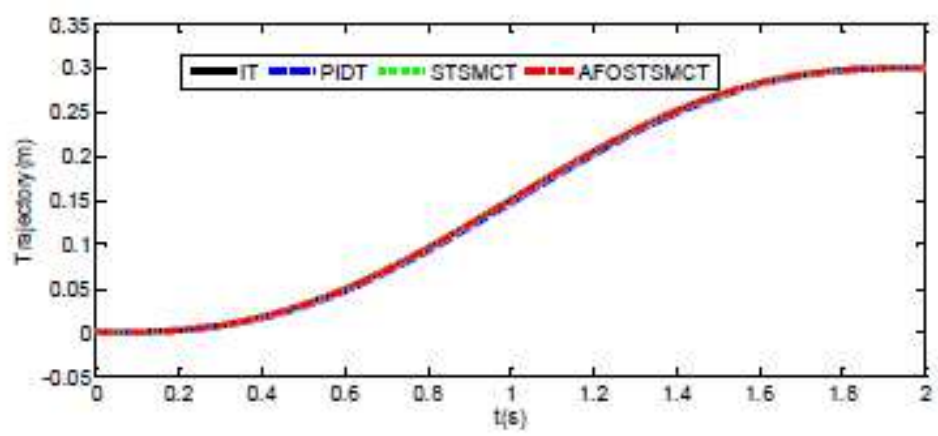

(a)

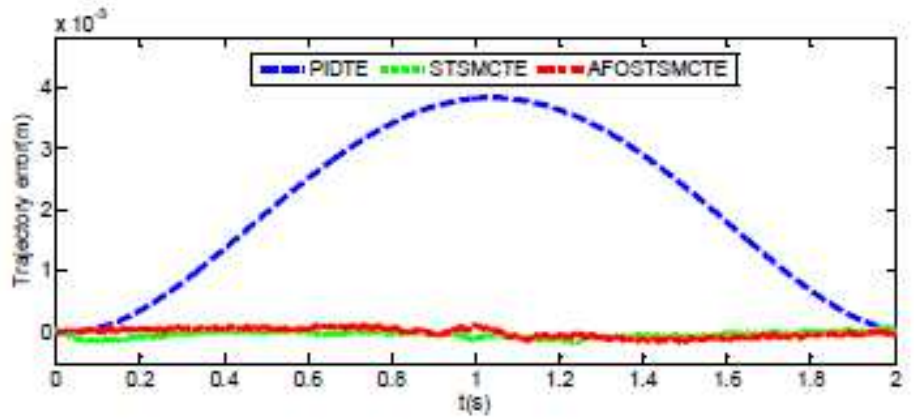

(b)

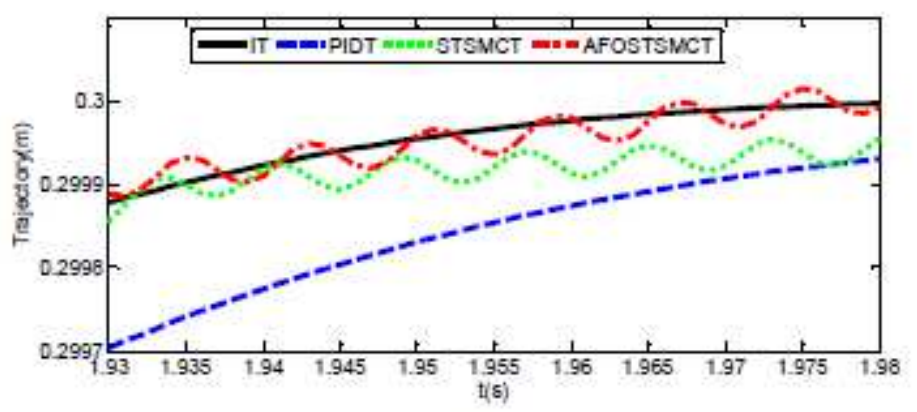

(c)

\section{Figure 3}

Trajectory tracking performance of the three controllers for the nominal model

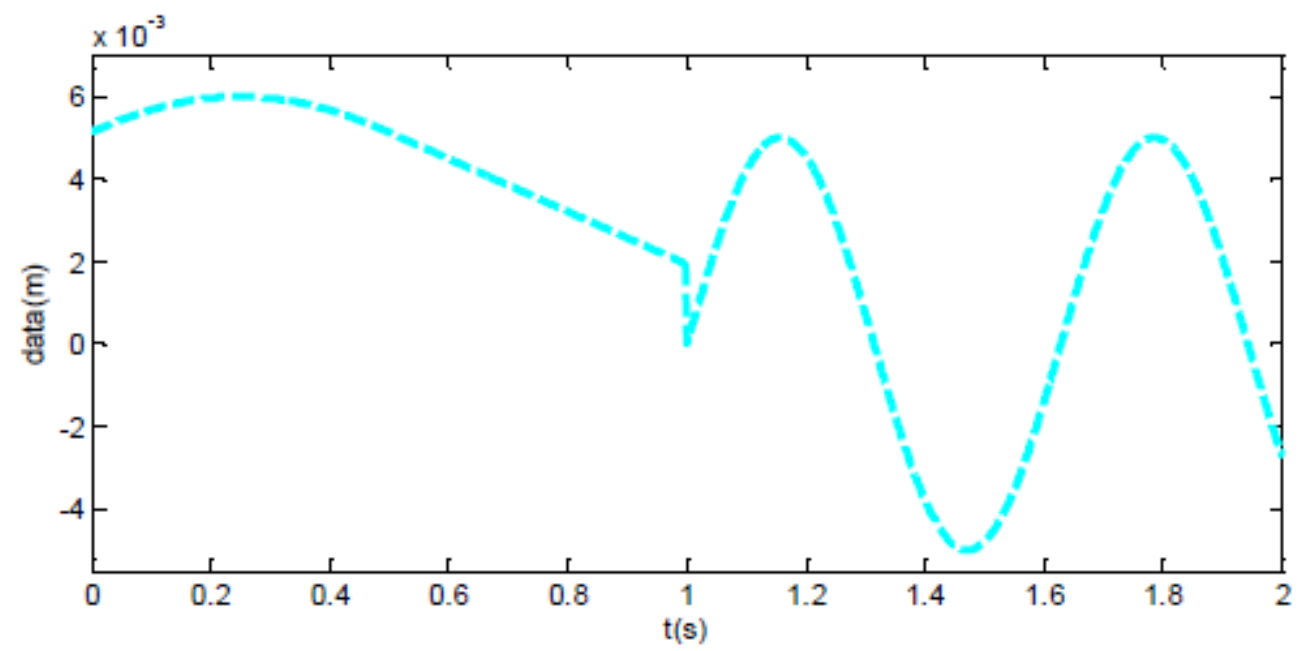


Figure 4

Position disturbance

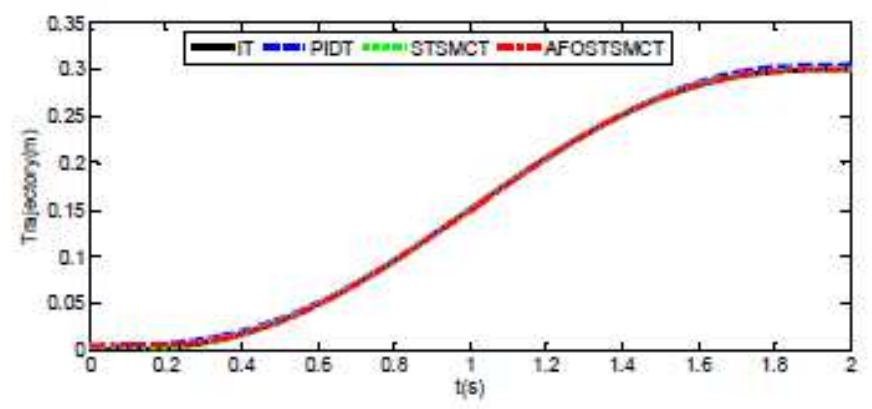

(a)

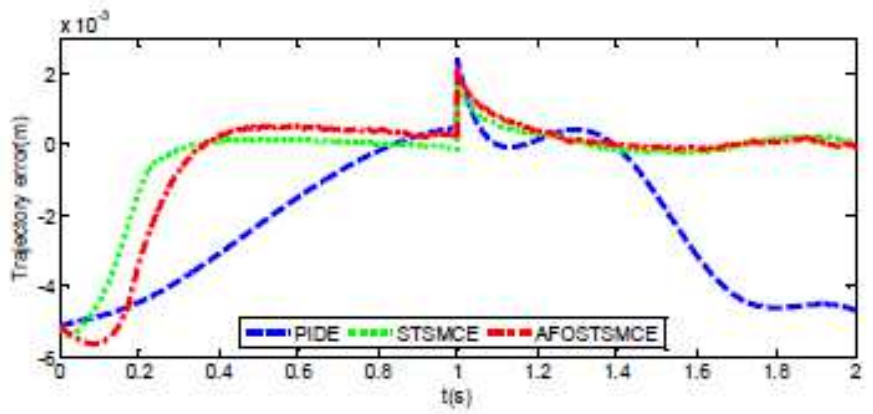

(b)

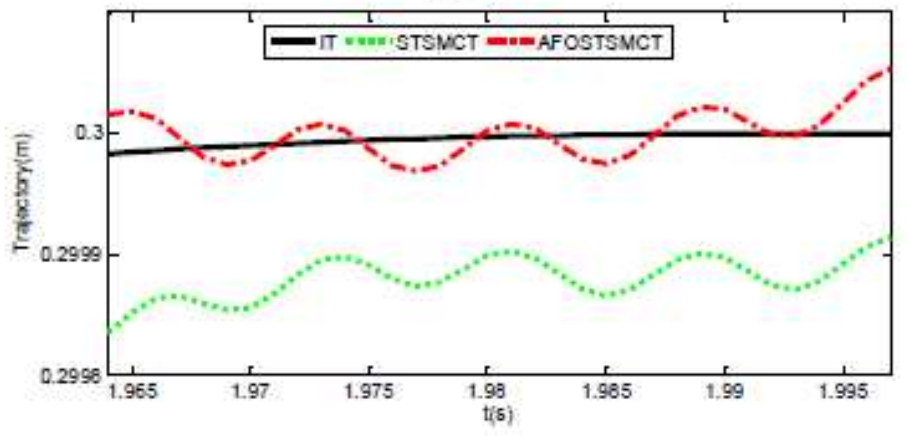

(c)

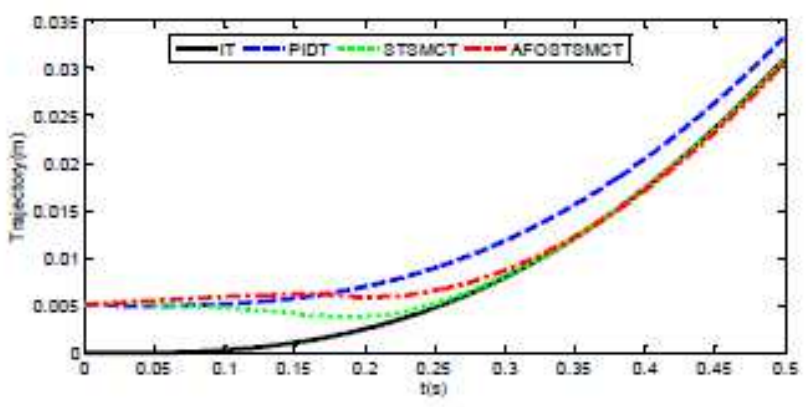

(d)

\section{Figure 5}

Trajectory tracking performance of the three controllers with position disturbance 